\title{
A retrospective cohort study comparing dairy calf treatment decisions by farm personnel with veterinary observations of clinical signs
}

\author{
A. Olson, ${ }^{1}$ W. M. Sischo, ${ }^{1}$ A. C. B. Berge,${ }^{2}$ A. Adams-Progar, ${ }^{3}$ and D. A. Moore ${ }^{1 *}$ \\ ${ }^{1}$ Department of Veterinary Clinical Sciences, Washington State University, Pullman 99164 \\ ${ }^{2}$ Department of Reproduction, Obstetrics and Herd Health, Faculty of Veterinary Medicine, Ghent University, 9820 Merelbeke, Belgium \\ ${ }^{3}$ Department of Animal Sciences, Washington State University, Pullman 99164
}

\section{ABSTRACT}

Antimicrobials are frequently administered to calves with diarrhea, despite evidence suggesting questionable efficacy. Even if efficacious, providing the appropriate therapy to an animal requires accurate disease detection. The objective of this study was to use previously collected data and compare clinical scoring by a veterinarian to treatment decisions by on-farm personnel. Data describing daily clinical scores and farm treatments were previously collected from 4 farms for calves from birth to age $28 \mathrm{~d}$. In this data set, a total of 460 calves were enrolled. Daily observations and clinical assessments were made on each farm by the same veterinarian, for a total of 12,101 calf observation days. Farm personnel made all treatment decisions based on their own observations, and these treatments were recorded by study personnel. Overall, the cumulative incidence of a calf exhibiting at least one abnormal clinical sign over the 28-d observation period was 0.93 , with cumulative incidences of 0.85 and 0.33 for diarrhea and dehydration, respectively. The cumulative incidence of any treatment (including antibiotics and electrolytes) was 0.85 , although the majority of treatments used an antimicrobial. The farm-specific probabilities that a calf with clinical signs of dehydration or diarrhea, respectively, received fluid or electrolyte therapy ranged from 0.08 to 0.27 and 0.03 to 0.12 . These probabilities were greater for the day a clinical sign was first observed. The farm-specific probabilities that a calf with clinical signs of diarrhea received an antimicrobial was 0.23 to 0.65 , and the probability that a calf exhibiting clinical signs of respiratory disease received an antimicrobial was 0.33 to 0.76 . The first observation of diarrhea had similar probabilities to those for all observations of diarrhea. There was greater probability of treatment for calves with their first observed ab-

Received August 29, 2018.

Accepted March 4, 2019.

*Corresponding author: damoore@wsu.edu normal respiratory signs. Probabilities that treatment with antimicrobials, or fluids or electrolytes, was associated with an abnormal clinical sign were low - that is, calves received treatments in the absence of any abnormal clinical signs. This study illustrates incongruity between treatment decisions by calf treaters (the designated personnel on each farm responsible for calf health assessment and treatment decisions) and those of an observer using a clinical scoring system to identify calves with abnormal clinical signs. These findings indicate opportunities and the need for dairy farmers and advisors to evaluate calf treatment protocols, reasons for treatment, and training programs for calf health and disease detection, as well as to develop monitoring programs for treatment protocol compliance and health outcomes following therapy.

Key words: dairy calf, diarrhea, respiratory disease, treatment

\section{INTRODUCTION}

Disease is commonly reported as a problem in the management of preweaned dairy calves. A USDA (2018) national study of US dairy operations reported that $21 \%$ of preweaned dairy calves were affected by diarrhea or other digestive disorders, and $76 \%$ of these calves were treated with an antibiotic. A survey of calf-raising facilities reported the median farm-level percentage of preweaned calf diarrhea to be $20 \%$ (interquartile range $=26.9 \%, \mathrm{n}=78$ ), and a median of $50 \%$ of all premise-level calf deaths were attributable to diarrhea (Walker et al., 2012). At the premise level, the median diarrhea-associated antibiotic treatment percent was $83 \%$ (interquartile range $=70 \%, \mathrm{n}=$ 73). These data highlight an important issue for the food-animal industries, including dairy and veterinary medicine: the use of antimicrobials for the management of calf health is common, and understanding how, why, and where antimicrobials are used is fundamental to developing, promoting, and fostering antibiotic stewardship (AVMA, 2018). 
Although the use of antibiotics to treat preweaned calf diarrhea is common and well documented, Smith (2015) raises a legitimate concern about their use. A very limited number of antimicrobials are approved to treat calf diarrhea (based on label claims), yet the data supporting the treatment efficacy of these labeled antimicrobials is inconsistent. In addition, although the industry has collected data describing how often antimicrobials are used, we know very little about the on-farm decision processes regarding calf health (such as what signs or cues define a calf as healthy or sick) or about the decision processes for intervening and treating (for instance, whether a calf receives supportive care or an antimicrobial).

Clinical scoring systems for diarrhea, dehydration, and respiratory disease have been developed to perform systematic assessments of calves to detect and rank severity of clinical disease (Fecteau et al., 1997; McGuirk, 2008). These tools can inform treatment decisions, support judicious use of antimicrobials, and improve calf health. Combining these scoring systems with important clinical signs such as attitude, appetite, swollen joints, ear droop, and navel swelling is effective in staging the health of a calf. In a study where the investigator based calf health assessments and antimicrobial treatment decisions upon a set of clinical scoring systems that included signs of fever, depression, or poor appetite, a substantial decrease in antimicrobial use and concomitant savings in drug costs were realized without affecting the farm's historic low mortality rates or targeted weight gains (Berge et al., 2009b). Although clinical scoring systems are prevalent in research studies, it is not known how commonly they are used on commercial dairy farms or calf ranches.

In a perfect world, all health and treatment decisions and associated treatment protocols would be evidencebased. However, farmers' and animal health workers' behaviors and decision-making are complex and based on intrinsic sociopsychological factors that are difficult to measure and predict. A study of dairy farmers' willingness to restrict use of anthelmintic drugs to reduce drug resistance revealed that their "attitude toward a novel treatment" and the "opinion of their significant other" had the strongest influence on adoption of restricted drug use (Vande Velde et al., 2015). Another study estimated that 17 to $47 \%$ of the variation in mastitis detection could be explained by a farmer's attitude (Jansen et al., 2009). A study of producer diagnosis of diarrhea in piglets found that producers with less than 4 years of experience were more accurate at diagnosing diarrhea than were producers with more than 4 years of experience (Pederson and Strunz, 2013). If level of experience, attitude, and influence by family members can have significant effects in other areas of agriculture, it seems that similar factors might play a role in dairy calf disease detection and treatment decisions.

Studies of disease diagnosis by producers, relative to veterinarian or diagnostic findings, have revealed discrepancies. Sivula et al. (1996) reported low sensitivity $(\mathrm{Se}=58 \%)$ and good specificity $(\mathrm{Sp}=93 \%)$ when comparing farmers' diagnoses of enteritis-associated calf mortality with necropsy findings. Producers' diagnoses of pneumonia-associated mortality were similarly aligned with necropsy findings $(\mathrm{Se}=56 \%$ and $\mathrm{Sp}=$ $100 \%$ ). A multifarm study comparing veterinary onfarm diagnosis of calf pneumonia (based on a set of clinical criteria) with treatment by on-farm personnel, based on their experiential criteria, reported that the farm personnel estimate of pneumonia population risk was 0.16 compared with the clinician estimate of 0.26 (Virtala et al., 1996a). Agreement between the groups was low (Kappa $=0.24$ ), and the discrepancy was attributed to underdetection of subclinical pneumonia by caregivers. In a companion paper comparing estimates of diarrhea risk on the same herds, Virtala et al. (1996b) reported their impression that caregiver treatment decisions overdiagnosed diarrhea compared with veterinary diagnosis based on clinical signs. In a study of morbidity in calves, Svensson et al. (2003) found that farmers were underdiagnosing respiratory disease compared with veterinarians who visited the farm monthly.

As the structure of US dairy and calf-rearing enterprises continues to move toward larger and more specialized operations, health care and treatment decisions are increasingly put in the hands of employees. In this context, we know relatively little about the relationship of employee decisions to treat a calf compared with daily clinical scoring of calf disease. The objective of this project was to compare clinical scoring of preweaned dairy calves by a veterinarian with treatment decisions by farm personnel and to describe on-farm treatment practices for calf health conditions.

\section{MATERIALS AND METHODS}

\section{Study Design and On-Farm Procedures}

For the purpose of this retrospective cohort study, data were sourced from previously conducted clinical trial studies, and specific details of farm management are described by Berge et al. (2009a,b). Briefly, data from 4 farms that rear calves were included. Three farms were calf ranches (CR1, dairy-source bull calves; CR2, dairy-source bull and heifer calves; CR3, dairysource bull calves), and during the study these farms 
enrolled 90, 84, and 95 calves, respectively. The fourth was a dairy farm (DF) raising their own heifer and bull calves; this farm enrolled 191 calves during the study. In these studies, all calves placed in the calf housing area were enrolled at birth (DF) or upon their arrival on the farm (CR1, CR2, CR3) and evaluated daily for up to $28 \mathrm{~d}$. Blood samples were collected on d 2 of life and evaluated for serum IgG using a radial immunodiffusion assay commercial kit (Immunocheck, VMRD, Pullman, WA). For this study, calves with values below $1,000 \mathrm{mg} / \mathrm{dL}$ were classified as failure to transfer passive immunity (FTPI). Clinical scores were assigned by a veterinary observer based on a multisymptom scoring system (Berge et al., 2005). Health parameters evaluated and scored included appetite (based on liquid feed consumption), fecal consistency, hydration, respiratory disease signs, and observations of eyes, ears, navel, joints, and calf attitude (Table 1). For analyses, all scores were dichotomized. Specifically, fecal clinical scores $0-1$ were classified as "normal," and scores of 2-4 classified as "diarrhea." Calf hydration score 0 was classified as "normal," and scores of 1-3 were classified as "dehydrated." Respiratory disease was classified as "normal" in the absence of clinical signs and classified as "diseased" with any sign of rhinitis, coughing, or heavy thoracic or abdominal breathing, and attitude was dichotomized as "alert" or "depressed." Calves were scored twice daily before milk feeding.

Table 1. Clinical scoring system used as criteria for defining disease in preweaned calves

\begin{tabular}{|c|c|c|}
\hline Observation & Clinical signs & Score \\
\hline \multirow[t]{5}{*}{ Fecal score } & Formed & 0 \\
\hline & Semi-formed/soft & 1 \\
\hline & Runny & 2 \\
\hline & Watery & 3 \\
\hline & Runny/watery with fecal blood & 4 \\
\hline \multirow[t]{5}{*}{ Respiratory } & Normal & 0 \\
\hline & Rhinitis (nasal discharge) & 1 \\
\hline & Coughing & 2 \\
\hline & Heavy thoracic breathing & 3 \\
\hline & Abdominal breathing & 4 \\
\hline \multirow[t]{4}{*}{ Hydration } & Normal appearance & 0 \\
\hline & Sunken eyes & 1 \\
\hline & Skin tented $5-10 \mathrm{~s}$ & 2 \\
\hline & Skin tented $>10 \mathrm{~s}$ & 3 \\
\hline \multirow[t]{3}{*}{ Attitude } & Alert & 0 \\
\hline & Depressed & 1 \\
\hline & Nonresponsive & 2 \\
\hline \multirow[t]{2}{*}{ Eyes } & Normal & 0 \\
\hline & Swelling/redness/discharge & 1 \\
\hline \multirow[t]{2}{*}{ Ears } & Normal & 0 \\
\hline & Head tilt, hanging head & 1 \\
\hline \multirow[t]{2}{*}{ Navel } & Normal & 0 \\
\hline & Swelling/heat/pain & 1 \\
\hline \multirow[t]{2}{*}{ Joints } & Normal & 0 \\
\hline & Swelling/heat/pain/lameness & 1 \\
\hline Appetite & Finished 2-L a.m. liquid meal & Yes/No \\
\hline
\end{tabular}

Personnel responsible for treatments on each farm made health and treatment decisions independent of veterinary observations and without diagnostic information. On all farms, one person was designated as a "treater" or "calf manager" and was responsible for treatment decisions. In addition, a designated person on each farm helped with treatments (often responsible for older preweaned calves) and was the "treater" on days when the primary treater was off farm. On all farms, the daily work routine included prescribed times allocated for health assessments and treatments. During this time, the veterinary investigator followed the treater and recorded events while treatments were administered; farm records were not used. Administration of an oral electrolyte solution (OES) was noted at this time, but actual administration of OES was usually deferred until after the clinical observation rounds and passively delivered as a bottle between feedings, verified at the subsequent health evaluation round. Data on clinical observations and treatments for calves within the first $28 \mathrm{~d}$ of life were merged, resulting in 460 calf records and a total of 12,101 calf-days of observation.

\section{Data Management and Analysis}

All health and treatment data were recorded in a spreadsheet program (Excel, Microsoft Corp., Redmond, WA). Descriptive statistics for total observation days, mortality, and clinical events during the observation period were calculated using $\mathrm{R}$ (version 3.4.3, $\mathrm{R}$ Project for Statistical Computing). Several outcome metrics were of interest, including cumulative incidence of clinical events, treatments, and mortality; ratio of cumulative number of clinical events to cumulative calf observation days for the 28-d follow-up period (28DCumE); daily prevalence of clinical events or treatments; time to first clinical or treatment event; and probability of an event.

Cumulative incidence was determined as the number of calves first observed with an event, relative to the calves at risk. The 28D-CumE for a clinical event or treatment was calculated as the ratio of the number of clinical events across the 28-d follow-up period relative to the number of cumulative observed calf-days. In this calculation, all observed daily events (not just first observation of an event) were counted. Daily prevalence of an event explicitly allowed calves to contribute several observations across the entire 28-d follow-up period. This was calculated as the number of observed events relative to the total number of calves observed that day and resulted in 28 discrete prevalence estimates. Time to first event, either clinical sign or treatment, was determined and reported as median time and in- 
terquartile range. Specifically, time to first observation of treatment was also described, using Kaplan-Meier methods ( $\mathrm{R}$ packages survival and survminer). Logistic regression models were used to estimate odds ratios (OR) and probabilities of first treatments with either electrolytes or antibiotics, conditional on the presence of clinical signs, and to estimate probabilities of first clinical signs conditional on a treatment event. A set of mixed logistic regression models for calves evaluated daily across the entire 28-d follow-up period were used to model treatments conditional on clinical signs. Solutions for multivariable logistic regression and mixed model multivariable logistic regression were determined using $\mathrm{R}$ packages glm and lme4, respectively. Probabilities for an outcome and their $95 \%$ confidence intervals were determined from the logistic regression variable estimates stratified by farm and solved using a spreadsheet program.

\section{RESULTS}

\section{Veterinary-Observed Abnormal Clinical Scores}

Our analysis included 460 Holstein calves. The allfarm 28-d mortality proportion was 0.10 and varied between farms, ranging from 0.02 to 0.28 (Table 2). The percentages of calves with FTPI (serum IgG values $<1,000 \mathrm{mg} / \mathrm{dL}$ ) were $64.4,45.6$, and $75.0 \%$ in CR1, CR2, and CR3, respectively. The percentage of calves with FTPI was $13 \%$ for DF. Daily health information was recorded over 12,101 calf observation days. Abnormal clinical scores were recorded on every farm. A total of 426 of 460 calves (92.6\%) exhibited at least one abnormal score during their 28-d follow-up. The 28D-CumE for abnormal signs observed on each farm and overall is shown (Table 2). Although some of the 28D-CumE were similar between study farms, farms had unique disease patterns.

First observations of abnormal clinical scores occurred during the first $3 \mathrm{~d}$ on CR1, CR2, and CR3, and primarily after d 9 on DF. The median days to observe the first sign were $3,1,3.5$, and $11 \mathrm{~d}$, with interquartile ranges of $4,6,5$, and 4 for CR1, CR2, CR3, and DF, respectively.

Diarrhea was the most common abnormal clinical score across all farms, with 393 of 460 calves (cumulative incidence $=0.85$ ) observed with at least one day of diarrhea and farm-specific diarrhea cumulative incidences of 0.9 (81/90), 0.75 (63/84), 0.73 (69/95), and 0.94 (179/191) for CR1, CR2, CR3, and DF, respectively. Diarrhea was also the most commonly observed first abnormal clinical score and accounted for 324 of the 426 observations. Across all observed calf-days, normal fecal scores (FS) were noted on 10,832 d and diarrhea observed 1,269 d (Table 2). The 28D-CumE for diarrhea was 0.10 , with farm-specific values ranging from 0.07 to 0.12 (Table 2). Based on the all-farm daily diarrhea prevalence, the interquartile range (i.e., half of the prevalence density) occurred in calves between ages 7 and $14 \mathrm{~d}$ (Figure 1). The temporal pattern for daily observations of diarrhea varied between farms. DF had a unimodal pattern, but CR1 and CR2 had bimodal patterns with peaks at d 1-3 and subsequent sets of observations uniformly distributed. However, daily diarrhea prevalence on CR2 persisted throughout the 28-d follow-up. For CR3 we discovered a fairly uniform pattern of daily diarrhea prevalence through d 9 and relatively low prevalence throughout the remaining follow-up (Figure 1). The overall median time to first observation of diarrhea was $9 \mathrm{~d}$, with an interquartile

Table 2. Summary of overall 28-d mortality and morbidity ${ }^{1}$

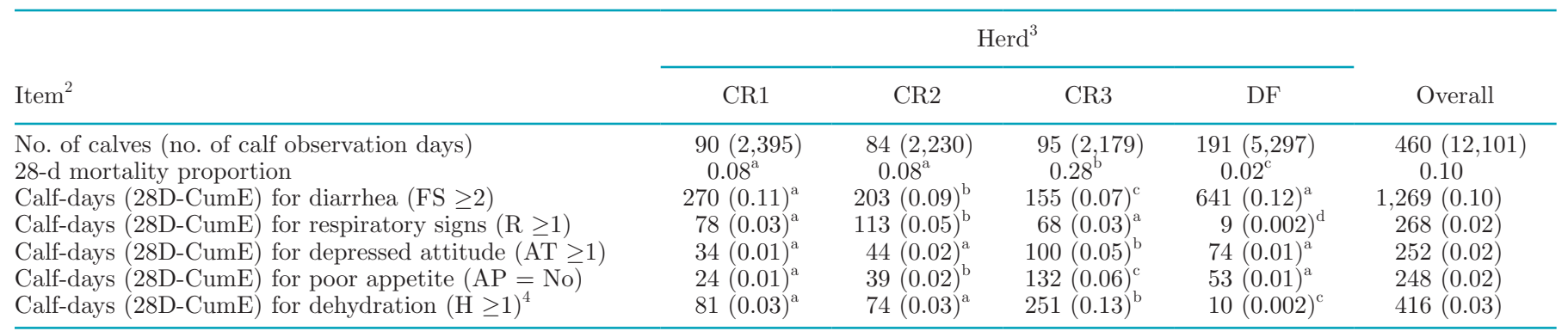

${ }^{\mathrm{a}-\mathrm{d}}$ Proportions within a row with different superscripts were statistically different $(P<0.05)$.

${ }^{1}$ Morbidity is based on daily veterinary clinical observations. Data are stratified by clinical sign and farm.

${ }^{2} \mathrm{FS}=$ fecal score; $\mathrm{R}=$ respiratory; $\mathrm{AT}=$ attitude; $\mathrm{AP}=$ appetite (finished a.m. liquid meal); $\mathrm{H}=$ hydration. $28 \mathrm{D}-\mathrm{CumE}$ was the ratio of cumulative number of observed clinical events to cumulative calf observation days for the 28-d follow-up. A calf observed multiple days with an event, for example, diarrhea, would be counted multiple times with that event.

${ }^{3} \mathrm{CR} 1, \mathrm{CR} 2, \mathrm{CR} 3, \mathrm{DF}=$ calf ranches 1,2 , and 3 , and dairy farm, respectively.

${ }^{4} 90 \%$ of calves were scored as $\mathrm{H}=1$ (sunken eyes). 


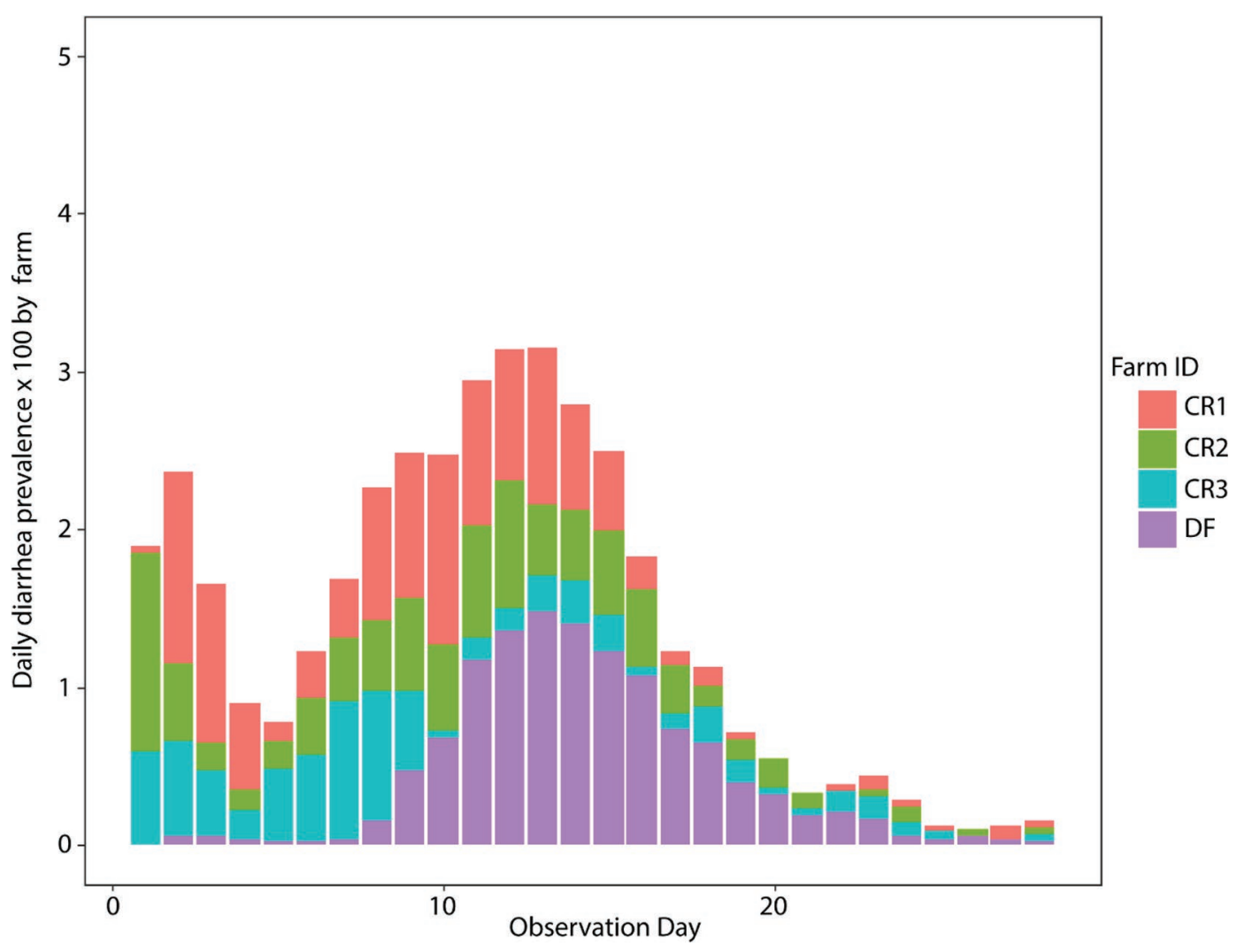

Figure 1. Daily prevalence of observed calf diarrhea stratified by farm across all observation days. Prevalence was calculated as daily cases of diarrhea relative to the total number of calves observed each day by farm and multiplied by 100 . CR1, CR2, CR3, DF $=$ calf ranches 1,2 , and 3 , and dairy farm, respectively.

range of $9 \mathrm{~d}$. For the calf ranches, the median day to first observation of diarrhea occurred within the first week (d 3,2, and 5), with an interquartile range of 6 , 8, and $6 \mathrm{~d}$ for CR1, CR2, and CR3, respectively. In contrast, the median day to first diarrhea observation for DF was $11 \mathrm{~d}$, with an interquartile range of $3 \mathrm{~d}$.

The second most common abnormal clinical score was dehydration. Across all farms, 150 out of 460 calves (cumulative incidence $=0.33$ ) were observed with at least one day of dehydration. This varied by farm, with cumulative incidences of 0.38 (34/90), 0.44 $(37 / 84), 0.74$ (70/95), and 0.05 (9/191) for CR1, CR2, CR3, and DF, respectively. For 65 calves, dehydration was the first observed abnormal score $(15 \%$ of first observations). The overall 28D-CumE for dehydration was $0.03(416 \mathrm{~d} / 12,101$ observation days), with farmspecific 28D-CumE ranging from 0.002 to 0.13 (Table 2 ). Based on the overall daily dehydration prevalence, the interquartile range was between 7 and $17 \mathrm{~d}$, with no clear peak day of occurrence (Figure 2). Farm CR3 was responsible for $62 \%$ of the cumulative daily dehydration events $(11.5 / 18.4$ dehydration events $\times 100)$ and consistently had clinical scores of dehydration across the 28-d follow-up period, while DF had few calves observed with dehydration (Figure 2). The overall median time to observe a first dehydration event was $8 \mathrm{~d}$, with an interquartile range of $8 \mathrm{~d}$. The patterns of first observation varied somewhat by farm, with median values of $8,14,7$, and 17 , and interquartile ranges of $2,9,8$, and 7 for CR1, CR2, CR3, and DF, respectively.

The remaining abnormal clinical observations (respiratory, attitude, appetite) were observed approximately $250 \mathrm{~d}$ each, for a 28D-CumE of 0.02 (Table 2). Across all observed abnormal clinical scores we observed a consistent pattern, with the 28D-CumE for farm DF below the overall value (the exception being diarrhea) and the 28D-CumE for farm CR3 above the overall value (the exception being diarrhea; Table 2).

Approximately $9 \%$ of calves observed with diarrhea on any day were also observed as clinically dehydrated, compared with $3 \%$ of calves with normal FS. Approximately $20 \%$ of dehydrated calves were also observed with a depressed attitude, compared with $2 \%$ of hydrated calves. 


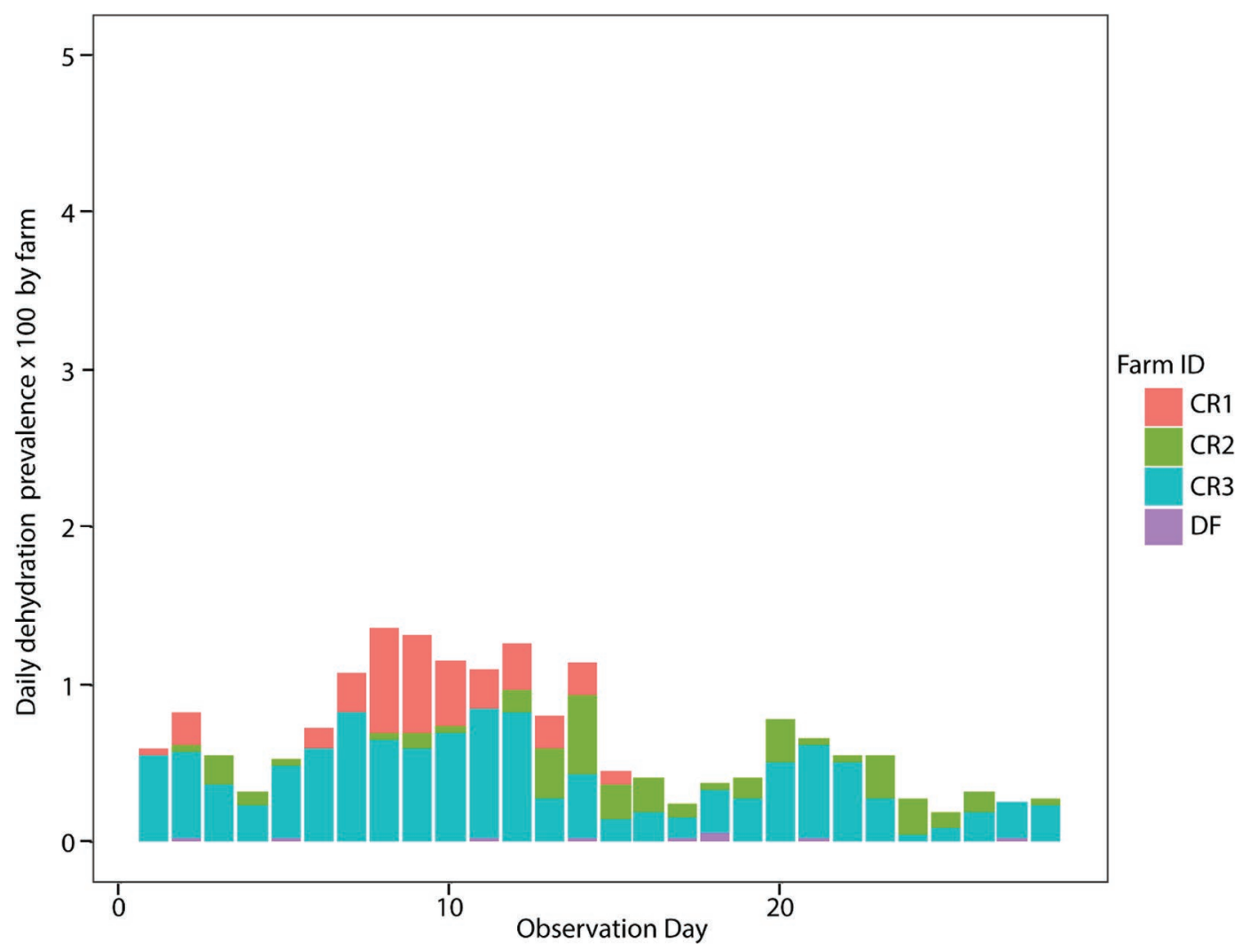

Figure 2. Daily prevalence of observed dehydration stratified by farm across all observation days. Prevalence was calculated as daily cases of dehydration relative to the total number of calves observed each day by farm and multiplied by 100 . CR1, CR2, CR3, DF $=$ calf ranches 1 , 2 , and 3 , and dairy farm, respectively.

\section{Antimicrobial and Non-Antimicrobial Treatments by On-Farm Personnel}

A total of 390 of the 460 calves $(84.7 \%)$ were treated at least once by farm personnel during their 28-d follow-up period. This included any administration of an antimicrobial, oral or intravenous fluids, an anti-inflammatory, or other palliatives, although $83 \%$ of first treatments were antimicrobials. Time to first treatment had 2 patterns, with similar median days at first treatment on the calf ranches: $5,5.5$, and $5 \mathrm{~d}$, with interquartile ranges of $8.25,14$, and $12 \mathrm{~d}$ for CR1, CR2, and CR3, respectively. Calves in DF had a later median of $11 \mathrm{~d}$ to first treatment, with a tight interquartile range of $3 \mathrm{~d}$. A Kaplan-Meier time-to-event curve demonstrates the same trend and also shows that by the end of the 28-d follow-up, nearly all calves on CR3 and DF had been treated, but slightly less than $75 \%$ of calves on CR1 and CR2 had been treated (Figure 3). By d 18, more than $96 \%$ of the treated cohorts for farms CR1, CR3, and DF had been treated. The survival curve for days to first treatment for CR2 had a more gradual slope, and $20 \%$ of first treatments had yet to be administered by d 18 (Figure 3 ).
Based on the daily prevalence of treatment, the interquartile range (i.e., $50 \%$ of daily treatments administered) was between 9 and $18 \mathrm{~d}$ (Figure 4). The temporal patterns of daily treatment prevalence varied, with the calf ranches treating in a more even pattern across the follow-up period and DF having a set of days with clear higher prevalence. The interquartile ranges for treatment overlapped between farms: $\mathrm{d} 6$ to 13, 8 to 20, 7 to 16 , and 8 to 18 for CR1, CR2, CR3, and DF, respectively.

The overall 28D-CumE for a calf to receive an antimicrobial treatment was 0.25 (Table 3). On more than $10 \%$ of calf-days with a FS $=0(1,118$ antibiotic treatment days $/ 9,223$ calf-days) and $15 \%$ of calf-days with FS $=1(237 / 1,609)$, calves were treated with an antimicrobial. Although all farms used 4 to 6 different antimicrobials in the first $28 \mathrm{~d}$ of life, the array of antimicrobials was unique to each farm. There was a between-farm difference in the number of observed days that antimicrobials were used. The greatest $28 \mathrm{D}$-CumE for antimicrobial treatments occurred at DF, which was mainly due to their use of a 2-antimicrobial protocol for the first treatment (Table 3 ). 
On 673 calf-days $(28 \mathrm{D}-\mathrm{CumE}=0.056)$ an $\mathrm{OES} / \mathrm{IV}$ fluid treatment was recorded, of which $13 \mathrm{~d}$ were IV fluids. Among the OES/IV fluid treatments, $75 \%$ were administered in conjunction with another treatment, and most of the associated treatments were antimicrobials (66.3\%). We observed farm differences, with DF having the lowest 28D-CumE for observed OES/ IV (0.024) compared with CR1, CR2, and CR3, which had 28D-CumE of 0.073, 0.073, and 0.095, respectively.

There were 187 recorded flunixin meglumine treatment days across the 4 farms. No specific clinical observations were associated with flunixin treatment, and antimicrobials were given at the same time on $85 \%$ of flunixin treatment days.

\section{Risk Factors for Electrolyte Treatments}

Using all observation days $(\mathrm{n}=12,101)$, from a mixed model logistic regression (controlling for farm and repeat observations on calves), we estimated OR and probabilities of OES/IV fluid treatment conditional on observing abnormal clinical signs associated with diarrhea, dehydration, poor appetite, and abnormal respiratory signs. Analysis revealed an increased likelihood that a calf with any symptom received an OES/IV fluid treatment compared with asymptomatic calves. The largest OR for receiving an OES/IV fluid treatment, compared with asymptomatic calves, were associated with observed dehydration (16.21, 95\% CI 12.01-22.42) and observed diarrhea (5.85, 95\% CI 4.69-7.30). These findings are tempered by the low probabilities of treating dehydrated calves and calves with diarrhea with OES/IV fluids. Similar probabilities $(\approx 0.26)$ of treating dehydrated calves with fluids occurred for CR1, CR2, and $\mathrm{CR}$, and DF was the least likely (probability 0.08) to treat with OES/fluids when the clinician observed clinical signs of dehydration only (Table 4). A similar pattern for farms was observed for calves with diarrhea, although these calves were very unlikely to receive an OES/IV treatment, with the probability of receiving fluids being approximately half of that for dehydrated calves (Table 4).

Logistic regression models for OES/IV treatment at first observation of dehydration or diarrhea also showed an increased risk for dehydrated calves to receive fluids $(\mathrm{OR}=20.46,95 \%$ CI 7.86-58.25). In contrast to the results using the full set of calf-day observations, the OR for calves with first day of observed diarrhea to receive fluids was diminished $(\mathrm{OR}=1.97,95 \%$ CI $0.83-4.85)$. However, these findings are also tempered by the low probabilities for calves to receive electrolytes, ranging from 0.18 to 0.59 for calves first observed as dehydrated and 0.02 to 0.12 for calves first observed with diarrhea (Table 5). It is notable that calves at first observation of dehydration were about 2 times more likely to receive an OES/IV treatment than were dehydrated

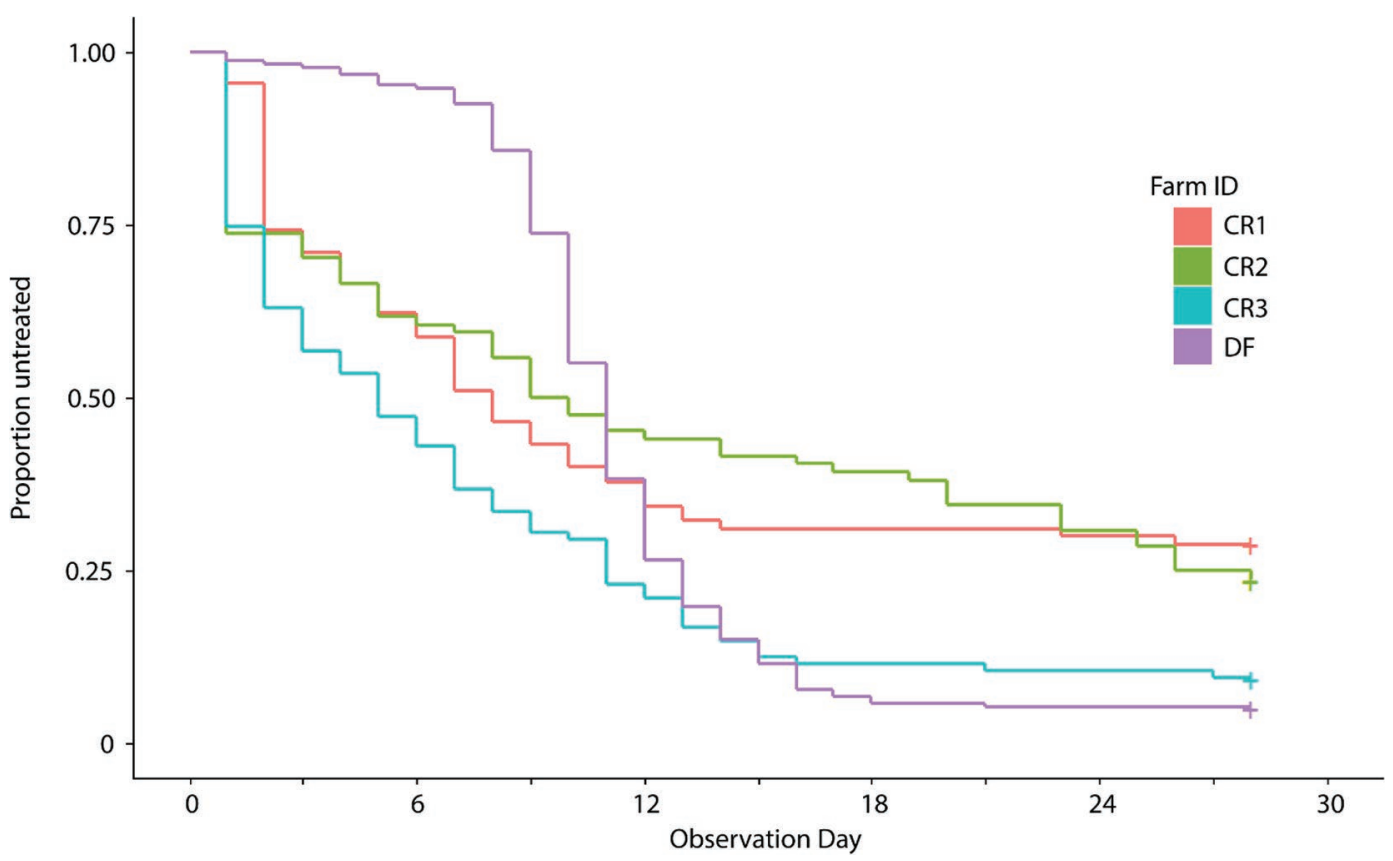

Figure 3. Kaplan-Meier plot of days to first treatment (electrolytes or antimicrobials) stratified by farm. CR1, CR2, CR3, DF $=$ calf ranches 1,2 , and 3 , and dairy farm, respectively. 
calves across the entire follow-up period. There was no such difference in probability for receiving fluid therapy between overall and first observation of diarrhea.

\section{Risk Factors for Antimicrobial Treatment}

Using all calf-day observations, from the results of a mixed model logistic regression controlling for farm and repeat observations of calves, we estimated OR and probability that a calf received an antimicrobial treatment if the calf was observed with diarrhea, did not finish its morning milk, exhibited respiratory signs, or was dehydrated compared with asymptomatic calves. All calves with any observed abnormal clinical signs were at increased risk for antimicrobial treatment compared with healthy calves, although the largest OR for receiving an antimicrobial were associated with calves observed with diarrhea $(\mathrm{OR}=11.06,95 \%$ CI 9.54-12.84) and calves with respiratory signs $(\mathrm{OR}=18.48,95 \% \mathrm{CI}$ 11.36-30.04). The probabilities of a calf receiving an antimicrobial for either diarrhea or respiratory signs varied by farm from 0.23 to 0.76 , with DF consistently having the greatest probability of using an antimicro- bial (Table 6). From the logistic regression evaluating antimicrobial treatment on first abnormal clinical score for diarrhea, the OR for antimicrobial treatment was comparatively muted ( $\mathrm{OR}=2.92,95 \%$ CI $1.35-6.42)$, although calves with respiratory signs had a similar risk for treatment on first sign as for signs across the entire follow-up period ( $\mathrm{OR}=15.08,95 \%$ CI 4.90-51.28). The probabilities that a calf with first observed day of diarrhea received an antimicrobial varied by farm but were relatively low for the calf ranches (0.17-0.40), with DF having the highest probability (0.67) of treating calves first observed with diarrhea with an antimicrobial (Table 7). The probabilities that a calf with first day of respiratory signs was treated with an antimicrobial were relatively high for all farms, with DF treating 0.91 of calves with signs (Table 7 ).

\section{Abnormal Clinical Observations Associated with First Treatments}

We evaluated a multivariable logistic regression model of the probability of observing a clinical sign, conditional on first treatment with an electrolyte or an

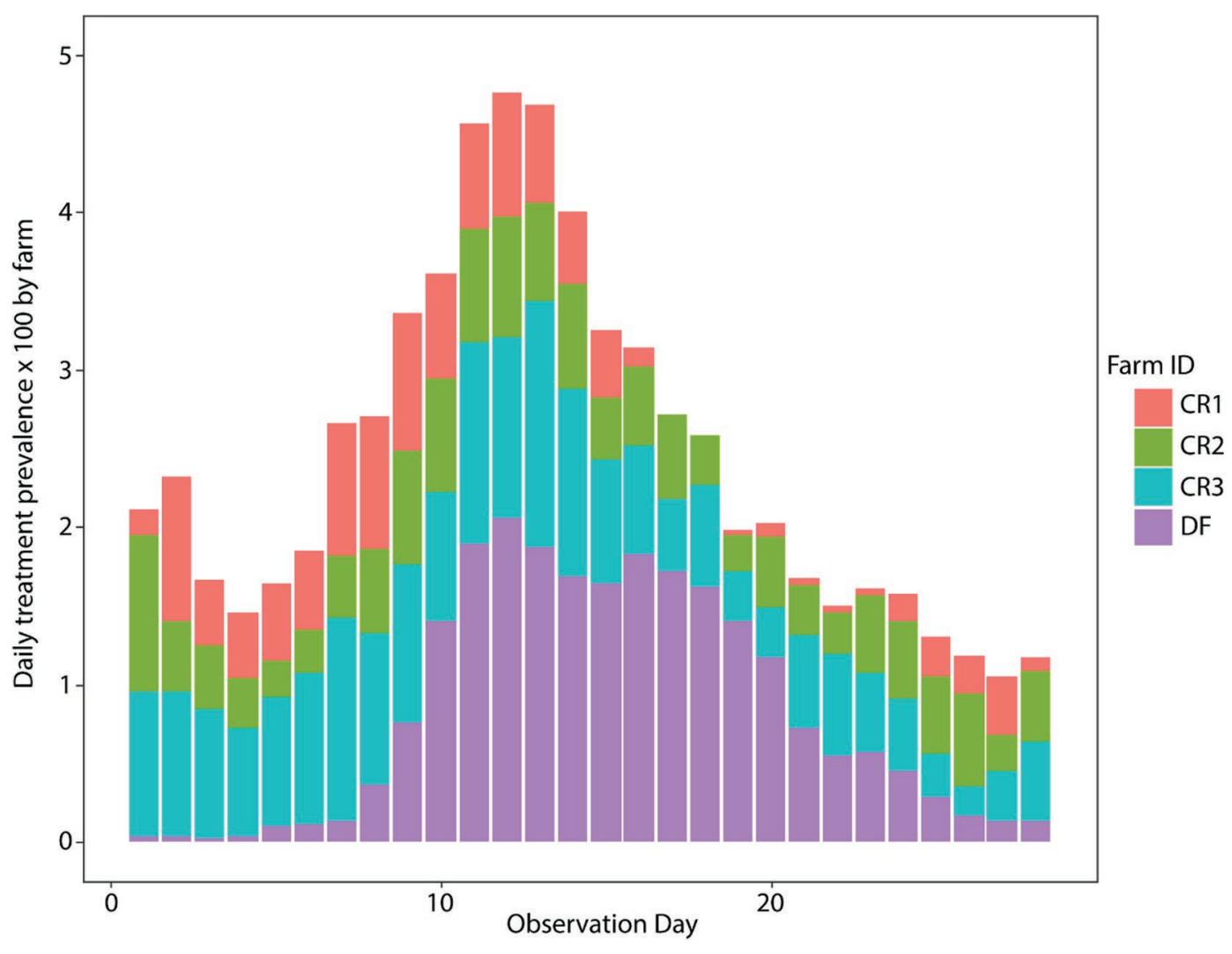

Figure 4. Daily prevalence of treatment stratified by farm across all observation days. Prevalence was calculated as number of daily treatments relative to the total number of calves observed each day by farm and multiplied by 100 . CR1, CR2, CR3, DF = calf ranches 1,2 , and 3 , and dairy farm, respectively. 
Table 3. Number of treatments (N; ratio of cumulative number of clinical events to cumulative calf observation days for the 28-d follow-up period in parentheses) stratified by antimicrobial and farm; data include 460 calves and 12,101 calf-days of observation

\begin{tabular}{|c|c|c|c|c|c|}
\hline \multirow[b]{2}{*}{ Antimicrobial } & \multicolumn{4}{|c|}{ Herd $^{1}$} & \multirow[b]{2}{*}{ Overall } \\
\hline & CR1 & $\mathrm{CR} 2$ & CR3 & $\mathrm{DF}$ & \\
\hline Penicillin & 0 & $17(0.0)$ & 0 & $633(0.12)$ & $650(0.05)$ \\
\hline Ceftiofur & $122(0.05)$ & $132(0.06)$ & $279(0.13)$ & 0 & $533(0.04)$ \\
\hline Florfenicol & 0 & $37(0.02)$ & $61(0.03)$ & $2(0.0)$ & $100(0.0)$ \\
\hline Fluoroquinolone & 0 & 0 & $1(0.005)$ & 0 & $1(0.0)$ \\
\hline Tilmicosin & $4(0.0)$ & $10(0.4)$ & 0 & 0 & $14(0.01)$ \\
\hline Tylosin & $18(0.0)$ & 0 & 0 & 0 & $18(0.0)$ \\
\hline Trimethoprim-sulfa & $65(0.03)$ & $83(0.04)$ & $49(0.02)$ & $828(0.16)$ & $1,025(0.08)$ \\
\hline Total & $216(0.09)$ & $283(0.13)$ & $390(0.18)$ & $2,096(0.4)$ & $2,985(0.25)$ \\
\hline Total observation days & 2,395 & 2,230 & 2,179 & 5,297 & 12,101 \\
\hline
\end{tabular}

${ }^{1} \mathrm{CR} 1, \mathrm{CR} 2, \mathrm{CR} 3, \mathrm{DF}=$ calf ranches 1,2 , and 3, and dairy farm, respectively.

antimicrobial, controlling for farm. There was a strong association for a fluid-treated calf to be observed as dehydrated $(\mathrm{OR}=13.5,95 \%$ CI $4.86-43.54)$ compared with a normal calf. The farm-specific probabilities of observing dehydration associated with a fluid treatment were variable and ranged between 0.12 and 0.62 , with CR3 having the greatest and DF the lowest probability (Table 8). We discovered no association for an antimicrobial-treated calf to be observed as dehydrated compared with a normal calf $(\mathrm{OR}=0.79,95 \%$ CI 0.36-1.73). The farm-specific probabilities reflected

Table 4. Probability of calf treatment by farm personnel, stratified by farm, with an oral electrolyte solution (OES) or IV fluids on any day given the clinical sign of dehydration only or diarrhea only, determined independently by a veterinarian ${ }^{1}$

\begin{tabular}{lcl}
\hline Herd & $\begin{array}{c}\text { Probability of } \\
\text { OES/fluid }\end{array}$ & $95 \%$ CI \\
\hline $\begin{array}{l}\text { Dehydration } \\
\text { CR1 }\end{array}$ & 0.27 & $0.18,0.37$ \\
CR2 & 0.26 & $0.18,0.37$ \\
CR3 & 0.26 & $0.17,0.36$ \\
DF & 0.082 & $0.057,0.12$ \\
Diarrhea & & \\
CR1 & 0.12 & $0.075,0.18$ \\
CR2 & 0.12 & $0.073,0.18$ \\
CR3 & 0.11 & $0.070,0.17$ \\
DF & 0.031 & $0.021,0.045$
\end{tabular}

${ }^{1}$ Probabilities estimated from mixed model logistic regression accounting for repeat observation of calves $(\mathrm{n}=460)$ during their first $28 \mathrm{~d}$ of life (12,101 total days of observation), conditional on clinical sign and farm

$\operatorname{Pr}$ OES/Fluid $=$

$1+e^{-[-5.2+1.41(\mathrm{CR} 1)+1.4(\mathrm{CR} 2)+1.35(\mathrm{CR} 3)+0(\mathrm{DF})+2.79(\mathrm{dehyd})+1.77(\text { dia })+1.15(\text { ap })+1.94(\text { resp })]}$

where presence of abnormal clinical scores, dehyd $=$ dehydration, dia $=$ diarrhea, $\mathrm{ap}=$ not completing a.m. milk, resp = respiratory disease, and $\mathrm{CR} 1, \mathrm{CR} 2, \mathrm{CR} 3, \mathrm{DF}=$ calf ranches 1,2 , and 3 , and dairy farm, respectively. this finding, as the probabilities for an antimicrobialtreated calf to be observed with dehydration were all below $10 \%$ (Table 8 ).

The results from a multivariable logistic regression model of the probability of observing diarrhea, conditional on a first fluid and antimicrobial treatment, found little evidence of an association between receiving a fluid treatment and being observed with diarrhea $(\mathrm{OR}=1.70,95 \%$ CI 0.90-3.24). The farm-specific probabilities for a fluid-treated calf observed with diarrhea ranged from 0.19 to 0.40 , with DF the most likely to have associated a fluid therapy with diarrheic calves (Table 9). There was a modest association between the

Table 5. Probability of calf treatment by farm personnel, stratified by farm, with an oral electrolyte solution (OES) or IV fluids on first observation of a clinical sign of dehydration only or diarrhea only, determined independently by a veterinarian ${ }^{1}$

\begin{tabular}{lcl}
\hline Herd & $\begin{array}{c}\text { Probability of } \\
\text { OES/fluid }\end{array}$ & $95 \%$ CI \\
\hline Dehydration & 0.59 & \\
CR1 & 0.59 & $0.32,0.86$ \\
CR2 & 0.40 & $0.33,0.86$ \\
CR3 & 0.18 & $0.16,0.74$ \\
DF & & $0.054,0.45$ \\
Diarrhea & 0.12 & $0.04,0.34$ \\
CR1 & 0.12 & $0.041,0.35$ \\
CR2 & 0.059 & $0.018,0.20$ \\
CR3 & 0.020 & $0.005,0.067$ \\
DF &
\end{tabular}

${ }^{1}$ Probabilities were estimated from a logistic regression accounting for clinical sign and farm:

$\operatorname{Pr}$ OES/Fluid $=$

$1+e^{-[-4.54+1.88(\mathrm{CR} 1)+1.89(\mathrm{CR} 2)+1.1(\mathrm{CR} 3)+0(\mathrm{DF})+3.029(\text { dehyd })+0.68(\text { dia })+0.1(\mathrm{ap})+1.8(\mathrm{resp})]}$,

where presence of abnormal clinical scores, dehyd = dehydration, dia $=$ diarrhea ap $=$ not completing a.m. milk, resp = respiratory disease, and CR1, CR2, CR3, DF = calf ranches 1, 2, and 3, and dairy farm, respectively. 
Table 6. Probability of calf treatment by farm personnel, stratified by farm, with an antimicrobial on any day given the clinical sign of diarrhea only or respiratory disease only, as determined independently by a veterinarian ${ }^{1}$

\begin{tabular}{lcc}
\hline & $\begin{array}{c}\text { Probability of } \\
\text { antimicrobial } \\
\text { treatment }\end{array}$ & $95 \%$ CI \\
\hline Herd & & \\
CR1 & 0.23 & $0.18,0.28$ \\
CR2 & 0.34 & $0.27,0.43$ \\
CR3 & 0.45 & $0.37,0.54$ \\
DF & 0.65 & $0.58,0.72$ \\
Respiratory disease & & \\
CR1 & 0.33 & $0.27,0.40$ \\
CR2 & 0.46 & $0.38,0.56$ \\
CR3 & 0.58 & $0.49,0.66$ \\
DF & 0.76 & $0.70,0.80$ \\
\hline
\end{tabular}

${ }^{1}$ Probabilities estimated from mixed model logistic regression, accounting for repeat observation of calves during their first $28 \mathrm{~d}$ of life $(12,101$ total days of observation), conditional on clinical sign and farm: Pr Antimicrobial Treatment $=$

1 $1+e^{-[-3.62+0(\mathrm{CR} 1)+0.55(\mathrm{CR} 2)+1.01(\mathrm{CR} 3)+1.82(\mathrm{DF})+1.25(\mathrm{dehyd})+2.4(\mathrm{dia})+1.59(\mathrm{ap})+2.92(\text { resp })]}$ where presence of abnormal clinical scores, dehyd $=$ dehydration, dia $=$ diarrhea, ap $=$ not completing a.m. milk, resp $=$ respiratory disease and $\mathrm{CR} 1, \mathrm{CR} 2, \mathrm{CR} 3, \mathrm{DF}=$ calf ranches 1,2 , and 3, and dairy farm, respectively.

first antimicrobial treatment and observing the treated calf with diarrhea $(\mathrm{OR}=3.22,95 \%$ CI $1.54-6.99)$. The farm-specific probabilities ranged from 0.31 to 0.56 (Table 9).

Table 7. Probability of calf treatment by farm personnel, stratified by farm, with an antimicrobial on first observation of a clinical sign of diarrhea only or respiratory disease only, determined independently by a veterinarian

\begin{tabular}{lcl}
\hline & $\begin{array}{c}\text { Probability of } \\
\text { antimicrobial } \\
\text { treatment }\end{array}$ & $95 \%$ CI \\
\hline Herd & & \\
CR1 & 0.17 & $0.069,0.36$ \\
CR2 & 0.40 & $0.24,0.59$ \\
CR3 & 0.23 & $0.12,0.38$ \\
DF & 0.67 & $0.51,0.80$ \\
Respiratory disease & & \\
CR1 & 0.58 & $0.28,0.74$ \\
CR2 & 0.77 & $0.62,0.88$ \\
CR3 & 0.60 & $0.40,0.76$ \\
DF & 0.91 & $0.84,0.95$ \\
\hline
\end{tabular}

${ }^{1}$ Probabilities were estimated from a logistic regression accounting for clinical sign and farm:

Pr Antimicrobial Treatment $=$

$1+e^{-[-2.64+0(\mathrm{CR} 1)+1.15(\mathrm{CR} 2)+0.35(\mathrm{CR} 3)+2.26(\mathrm{DF})+0.97(\mathrm{dehyd})+1.07(\mathrm{dia})+0.18(\mathrm{ap})+2.71(\text { resp })]}$

where presence of abnormal clinical scores, dehyd $=$ dehydration, dia

$=$ diarrhea, ap = not completing a.m. milk, resp = respiratory disease, and CR1, CR2, CR3, DF = calf ranches 1, 2, and 3, and dairy farm, respectively.
Table 8. Probability of observing a calf with dehydration only (determined independently by a veterinarian) stratified by farm, conditional on the first application of an electrolyte treatment or an antimicrobial treatment ${ }^{1}$

\begin{tabular}{lcl}
\hline & $\begin{array}{c}\text { Probability of } \\
\text { observing } \\
\text { dehydration }\end{array}$ & \\
\hline Electrolyte treatment & $95 \%$ CI \\
CR1 & 0.33 & $0.17,0.49$ \\
CR2 & 0.27 & $0.086,0.46$ \\
CR3 & 0.62 & $0.46,0.77$ \\
DF & 0.12 & $0,0.31$ \\
Antimicrobial treatment & & \\
CR1 & 0.028 & $0.00,0.060$ \\
CR2 & 0.021 & $0.00,0.047$ \\
CR3 & 0.082 & $0.010,0.16$ \\
DF & 0.0082 & $0.00,0.020$ \\
\hline
\end{tabular}

${ }^{1}$ Probabilities were estimated from a logistic regression:

Pr Dehydration $=$

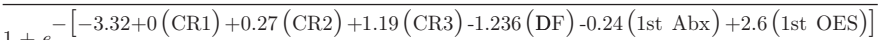
where $\mathrm{Abx}=$ antibiotic treatment, OES = electrolyte treatment, and $\mathrm{CR} 1, \mathrm{CR} 2, \mathrm{CR} 3, \mathrm{DF}=$ calf ranches 1,2 , and 3 , and dairy farm, respectively.

\section{DISCUSSION}

Our study, using previously collected data involving 4 commercial farms rearing dairy-source calves, found that an observation of an abnormal clinical sign or score by a veterinary observer was not consistently associated with a calf caretaker's decision to treat with either an antimicrobial or electrolytes or fluid (OES/ fluid). In addition, calf workers' decisions to treat a

Table 9. Probability of observing a calf with diarrhea only (determined independently by a veterinarian) stratified by farm, conditional on the first application of an electrolyte treatment or an antimicrobial treatment

\begin{tabular}{lcc}
\hline & $\begin{array}{c}\text { Probability of } \\
\text { observing } \\
\text { diarrhea }\end{array}$ & $95 \%$ CI \\
Herd & 0.25 & $0.12,0.38$ \\
Electrolyte treatment & 0.23 & $0.091,0.37$ \\
CR1 & 0.19 & $0.084,0.30$ \\
CR2 & 0.40 & $0.20,0.60$ \\
DF & & \\
Antimicrobial treatment & 0.39 & $0.23,0.54$ \\
CR1 & 0.36 & $0.22,0.51$ \\
CR2 & 0.31 & $0.19,0.44$ \\
CR3 & 0.56 & $0.49,0.64$ \\
DF &
\end{tabular}

${ }^{1}$ Probabilities were estimated from a logistic regression: Pr Diarrhea $=$

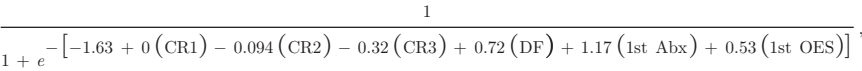

where $\mathrm{Abx}=$ antibiotic treatment, OES = electrolyte treatment, and CR1, CR2, CR3, DF = calf ranches 1,2 , and 3, and dairy farm, respectively. 
calf with an antimicrobial or OES/fluid were also not consistently associated with an abnormal clinical sign or score.

Although we observed variation among farms in the use of electrolytes, the consistent pattern was that all study farms used fluid treatment sparingly. The 28DCumE of a calf receiving an OES/fluid treatment was only 0.06 , and the overall $28 \mathrm{D}-\mathrm{CumE}$ of diarrhea or dehydration was 0.13 . The calf ranches had relatively similar use patterns, and DF rarely used OES/fluids. Although calves with abnormal clinical scores associated with dehydration or diarrhea were more likely to receive OES/fluid than normal calves, the probabilities were low that calves with those symptoms did receive fluids. Despite calves first observed with dehydration being more likely to receive an OES/fluid than for any observation, calves with first observation of diarrhea were no more likely to receive fluids than normal calves or for any observation of diarrhea. It was also true that calves receiving electrolyte therapy had low probabilities of being observed as dehydrated or with diarrhea. Given that the 28D-CumE of diarrhea or dehydration was 0.13 , and considering the importance of fluid therapy in correcting dehydration and metabolic imbalance associated with diarrhea (Smith and Berchtold, 2014), all farms had opportunities to increase treatment with electrolytes.

There was also variation among farms in the use of antimicrobials, but the consistent finding was that antimicrobials were used less frequently than would be indicated by observed abnormal clinical scores. The exception was DF, in that it overused antibiotics. The 28D-CumE for all abnormal clinical score risk was 0.19 for $\mathrm{DF}$, yet its 28D-CumE for antimicrobial use was 0.25 . This is mainly explained by DF's use of a 2-antibiotic combination therapy. Even though a calf with diarrhea (at any time or on first observation) was more likely to receive an antimicrobial treatment than an electrolyte, a high probability remained that a calf with diarrhea symptoms would not receive an antimicrobial. The probability was also high that calves receiving an antimicrobial had no recorded clinical sign of diarrhea. There was no difference in the probability of receiving an antimicrobial, whether it was first observation or any observation of diarrhea. Even though the use of antimicrobials was muted relative to the observation of abnormal clinical signs, a high proportion (84.7\%) of calves were treated with antimicrobials, and the mismatch between observed signs and treatment decisions suggests that we have much to understand about how, when, and why antimicrobials are administered to preweaned dairy calves. This information is necessary in order to implement judicious use practices on farms (AVMA, 2018).
We observed a similar pattern of probability for antimicrobial use to treat respiratory signs, although analysis revealed that the probability of treatment was greater on first observation than for any other observation. This may be associated with treatment protocols that do not have every-day treatment regimens. Even focusing on first observation of respiratory disease and assuming that first therapy is with an antimicrobial, a high proportion of calves with respiratory signs remained untreated, presumably because calf-care personnel did not recognize respiratory signs. Sivula et al. (1996) reported discrepancies between necropsy results and producer diagnoses of enteric and respiratory disease. Producers were only slightly better than a coin flip at diagnosing enteric- and respiratory-associated mortality. Svensson et al. (2003) reported that, compared with a veterinarian, producers underdiagnosed respiratory disease, which supports our finding that many calves with first observation of an abnormal respiratory score were left untreated.

In our study, we relied on a simple clinical scoring system to establish the health status of calves and compared these results to treatment decisions made by onfarm personnel (Berge et al., 2009b). Current evidence indicates that this system is a good reflection of calf health and should act as criteria for treatment decisions (McGuirk and Peek, 2014; Mahendran et al., 2017). When analyzing clinical signs associated with the first day of treatment, a high proportion of treated calves in our study had no abnormal clinical scores recorded associated with either dehydration or diarrhea. For respiratory disease, there was some discordance between signs observed and treatment decisions by farm staff. In a study of thoracic ultrasound of preweaned calves to identify respiratory disease, investigators found that $28 \%$ of calves had been treated but had no thoracic ultrasound lesions (Buczinski et al., 2014). This finding suggests several explanations for the discordance. First, the clinical scoring systems may be flawed and do not reflect risk of disease and other criteria being used for treatment decisions by on-farm calf-care personnel. A second explanation is that on some farms we may have failed to teach employees how to use clinical criteria to support treatment decisions. In previous work, we have shown that veterinarians have infrequently been involved in calf-care worker training and that most training occurs within the farm workforce, either worker-to-worker or manager-to-worker (Sischo et al., 2019). Either way, if treatment protocols include clear definitions of disease, particularly for diarrhea, calf-care workers might be better able to target appropriate interventions and reduce antimicrobial use. This requires veterinarians and producers to investigate whether calf caretakers are identifying clinical signs 
before treatment and on what criteria they are basing treatment decisions.

The wide variety of antimicrobials used for treatments, particularly diarrhea, indicates the need for data on efficacy of these different therapies and how efficacy may change based on varying clinical criteria. Because most of these antimicrobials lack on-label indications for treatment of diarrhea, off-label use of these drugs requires a prescription and protocol. Although veterinarians might provide a protocol for use of these drugs, it is possible for those responsible for treatment to use alternative strategies for the drugs, such as antibiotic "cocktails," or combinations of drugs, because of the perceived lack of efficacy when following protocols. Without knowledge of specific signs upon which to base treatments, as well as knowledge of what criteria could determine a successful treatment, treatment decisions may be based on beliefs of efficacy rather than science.

Although our study farms were not purposively selected as a reflection of the US dairy industry, these farms may reflect the diversity of clients of many veterinary practitioners and dairy consultants. Our study farms were heterogeneous; they had different mortality experiences during the times of observation, experienced different risks of FTPI, followed different strategies for treating similar health conditions, and faced different management challenges during the follow-up periods. As an example, the farm with the highest mortality during the study (CR3, $28 \%$ mortality in $28 \mathrm{~d}$ of observation) experienced a Salmonella outbreak during the observation period. In addition, the number of observed and recorded abnormal clinical scores differed across the study farms, as did the temporal patterns of when signs were observed. Despite these differences, the patterns of electrolyte and antimicrobial use were relatively similar across these farms.

The results reported from our study farms provide some insight on how some farms might approach implementing judicious use guidelines for treating preweaned calves: (1) Data summaries based on farm treatment information may not represent actual disease events; (2) the processes and criteria or cues for making treatment decisions may be variable among farms and likely reflect variability in decision processes among those responsible for treatment; (3) veterinarians and farmers should consider evaluating treatment decisions and protocol compliance; and (4) treatment protocols should include criteria for disease detection as well as the adjunct and effective therapies for clinical signs and most likely disease processes. All of these elements represent opportunities for education and training programs on disease detection, appropriate therapy, and appropriate health monitoring.

\section{CONCLUSIONS}

This study illustrates incongruity between treatment decisions by calf treaters and those of an observer using a defined set of clinical signs on 4 farms and highlights an opportunity for dairy farmers and advisors to evaluate calf treatment protocols, reasons for treatment, and training programs for calf health and disease detection, as well as to develop monitoring programs for treatment protocol compliance and health outcomes.

\section{ACKNOWLEDGMENTS}

Data were generated from a project funded by USDA Cooperative State Research, Education, and Extension Service National Integrated Food Safety Initiative 2004-51110-02184 and USDA National Institute for Food and Agriculture (Washington, DC; Grant No. 2015-68003-22998).

\section{REFERENCES}

American Veterinary Medical Association. Antimicrobial Stewardship Definition and Core Principles. 2018. Accessed Dec. 3, 2018. https: //www.avma.org/KB/Policies/Pages/Antimicrobial-Stewardship -Definition-and-Core-Principles.aspx.

Berge, A. C. B., T. E. Besser, D. A. Moore, and W. M. Sischo. 2009a. Evaluation of the effects of oral colostrum supplementation during the first fourteen days on the health and performance of preweaned calves. J. Dairy Sci. 92:286-295. https://doi.org/10.3168/ jds.2008-1433.

Berge, A. C. B., D. A. Moore, T. E. Besser, and W. M. Sischo. 2009b. Targeting therapy to minimize antimicrobial use in preweaned calves: Effects on health, growth, and treatment costs. J. Dairy Sci. 92:4707-4714. https://doi.org/10.3168/jds.2009-2199.

Berge, A. C. B., D. A. Moore, P. Lindeque, and W. M. Sischo. 2005. A clinical field trial in a calf ranch evaluating the influence of prophylactic and therapeutic antibiotic use on calf health. J. Dairy Sci. 88:2166-2177. https://doi.org/10.3168/jds.S0022-0302(05)72892 -7 .

Buczinski, S., G. Forté, D. Francoz, and A.-M. Bélanger. 2014. Comparison of thoracic auscultation, clinical score, and ultrasonography as indicators of bovine respiratory disease in preweaned dairy calves. J. Vet. Intern. Med. 28:234-242. https://doi.org/10.1111/ jvim.12251.

Fecteau, G., J. Paré, D. C. Van Metre, B. P. Smith, C. A. Holmberg, W. Guterbock, and S. Jang. 1997. Use of a clinical sepsis score for predicting bacteremia in neonatal dairy calves on a calf rearing farm. Can. Vet. J. 38:101-104.

Jansen, J., B. H. van den Borne, R. J. Renes, G. van Schaik, T. J. Lam, and C. Leeuwis. 2009. Explaining mastitis incidence in Dutch dairy farming: The influence of farmers' attitudes and behavior. Prev. Vet. Med. 92:210-223. https://doi.org/10.1016/j .prevetmed.2009.08.015.

Mahendran, S. A., R. Booth, L. Beekhuis, A. Manning, T. Blackmore, A. Vanhoudt, and N. Bell. 2017. Assessing the effects of weekly preweaning health scores on dairy calf mortality and productivity parameters: Cohort study. Vet. Rec. 181:196-202. https://doi.org/ 10.1136/vr.104197.

McGuirk, S. M. 2008. Disease management of dairy calves and heifers. Vet. Clin. North Am. Food Anim. Pract. 24:139-153. https://doi .org/10.1016/j.cvfa.2007.10.003. 
McGuirk, S. M., and S. F. Peek. 2014. Timely diagnosis of dairy calf respiratory disease using a standardized scoring system. Anim. Health Res. Rev. 15:145-147.

Pederson, K. S., and A. M. Strunz. 2013. Evaluation of farmers' diagnostic performance for detection of diarrhea in nursery pigs using digital pictures of fecal pools. Acta Vet. Scand. 55:72-77. https:// doi.org/10.1186/1751-0147-55-72. .

Sischo, W. M., D. A. Moore, R. Pereira, L. Warnick, D. L. Moore, J. Vanegas, S. Kurtz, K. Heaton, D. Kinder, J. Siler, and M.A. Davis. 2019. Calf care personnel on dairy farms and their educational opportunities. J. Dairy Sci. 102:3501-3511. https://doi.org/10.3168/ jds.2018-15401.

Sivula, N. J., T. R. Ames, W. E. Marsh, and R. E. Werdin. 1996. Descriptive epidemiology of morbidity and mortality in Minnesota dairy heifer calves. Prev. Vet. Med. 27:155-171.

Smith, G. 2015. Antimicrobial decision making for enteric diseases of cattle. Vet. Clin. North Am. Food Anim. Pract. 31:47-60. https:/ /doi.org/10.1016/j.cvfa.2014.11.004.

Smith, G. W., and J. Berchtold. 2014. Fluid therapy in calves. Vet. Clin. North Am. Food Anim. Pract. 30:409-427. https://doi.org/ 10.1016/j.cvfa.2014.04.002

Svensson, C., K. Lundborg, U. Emanuelson, and S. Olsson. 2003. Morbidity in Swedish dairy calves from birth to 90 days of age and individual calf-level risk factors for infectious diseases. Prev. Vet. Med. 58:179-197. https://doi.org/10.1016/S0167-5877(03)00046 -1 .
USDA. 2018. Health and Management Practices on U.S. Dairy Operations, 2014. CEAH $<$ National Animal Health Monitoring System, Fort Collins, CO. \#696.0218. Accessed Dec. 3, 2018. https://www .aphis.usda.gov/animal_health/nahms/dairy/downloads/dairy14/ Dairy14_dr_PartIII.pdf.

Vande Velde, F., E. Claerbout, V. Cauberghe, L. Hudders, and H. Van Loo. 2015. Diagnosis before treatment: Identifying dairy farmers' determinants for the adoption of sustainable practices in gastrointestinal nematode control. Vet. Parasitol. 212:308-317. https://doi .org/10.1016/j.vetpar.2015.07.013.

Virtala, A. M. G. D. Mechor, Y. T. Gröhn, and H. N. Erb. 1996a Epidemiologic and pathologic characteristics of respiratory tract disease in dairy heifers during the first three months of life. J. Am. Vet. Med. Assoc. 208:2035-2042.

Virtala, A. M., G. D. Mechor, Y. T. Gröhn, and H. N. Erb. 1996b. Morbidity from nonrespiratory diseases and mortality in dairy heifers during the first three months of life. J. Am. Vet. Med. Assoc. 208:2043-2046.

Walker, W. L., W. B. Epperson, T. E. Wittum, L. K. Lord, P. J. Rajala-Schultz, and J. Lakritz. 2012. Characteristics of dairy calf ranches: Morbidity, mortality, antibiotic use practices, and biosecurity and biocontainment practices. J. Dairy Sci. 95:2204-2214. https://doi.org/10.3168/jds.2011-4727. 